\title{
The time course of the BOLD response in the human auditory cortex to acoustic stimuli of different duration
}

\author{
L. Jäncke ${ }^{a, b, *}$, T. Buchanan ${ }^{\text {b,c }}$, K. Lutz ${ }^{\text {b }}$, K. Specht ${ }^{b}$, S. Mirzazade ${ }^{b}$, N.J.S. Shah ${ }^{b}$ \\ ${ }^{a}$ Institute of General Psychology, Otto-von-Guericke University Magdeburg, Magdeburg, Germany \\ ${ }^{\mathrm{b}}$ Institute of Medicine, Research Center Jülich, Germany \\ ${ }^{c}$ The University of Oklahoma, Health Sciences Center, Department of Psychiatry and Behavioral Sciences, Oklahoma City, OK, USA
}

Accepted 30 March 1999

\begin{abstract}
The relationship between activity within the human auditory cortices and the duration of heard tones was investigated by measuring the hemodynamic response with functional magnetic resonance imaging. We demonstrate that there is no significant influence of stimulus duration as used here on the intensity and spatial extent of the hemodynamic response in the auditory cortices. We found however, that the time course of the hemodynamic response to the repeated stimulus presentation exhibited a characteristic decline after the first stimulus exposure during the activation period. The possible reasons for this time course are currently unknown, however, several factors may be involved, including top-down mechanisms and/or the interplay of tissue perfusion and oxygen consumption. (C) 1999 Elsevier Science B.V. All rights reserved.
\end{abstract}

Keywords: fMRI; Auditory cortex; Stimulus duration

\section{Introduction}

The susceptibility of blood oxygenation level dependent (BOLD) effect can be exploited to provide activation maps of the human auditory cortex by MRI in response to acoustic stimuli $[6-8,10,11,29,33]$. Much of this work has proceeded with little information about the effects on response magnitude of such stimulus variables such as rate, intensity, and duration. Only a few studies investigated the influence of these basic stimulus variables on the BOLD response. For instance intensity, rate as well as the TR time have been shown to be significant variables determining the BOLD response in the primary and secondary auditory cortex $[3,4,11,24]$. However, stimulus duration and its potential influence on the BOLD response has not been investigated in detail. The present study therefore was designed to investigate the relationship between stimulus duration and the BOLD response in the

\footnotetext{
* Corresponding author. Institute of General Psychology, Otto-vonGuericke University Magdeburg, Lennéstraße 6, D-39112 Magdeburg, Germany. Fax: + 49-391-6714815; E-mail: lutz.jaencke@gse-w.uni-magdeburg.de
}

primary and secondary auditory cortex. Because a recent fMRI study suggested considerable spatio-temporal variability of the hemodynamic response to the same repetitive auditory stimulus [20], we also analysed the time course of the BOLD response within the primary and secondary auditory cortex in relation to stimulus duration.

\section{Materials and methods}

Five male right-handed volunteers, ranging in age from 20 to 40 years, with no history of neurological or audiological illness were studied. After a full explanation of the nature and risks of the research, subjects gave informed written consent for all studies according to a protocol approved by the Ethics Committee of the Heinrich-Heine University, Düsseldorf.

Functional MR images were acquired using a $1.5 \mathrm{~T}$ Siemens MRI system (SIEMENS Magnetom Vision, Erlangen), equipped with echo planar imaging (EPI) capability and a standard radiofrequency (RF) head coil for transmit and receive. Pulse sequence parameters were as follows: EPI; repetition time $(\mathrm{TR})=6 \mathrm{~s}$; echo time $(\mathrm{TE})=$ $66 \mathrm{~ms}$; field of view $(\mathrm{FOV})=200 \times 200 \mathrm{~mm}$; flip angle 
$(\alpha)=90^{\circ}$; matrix size $=64 \times 64$; in-plane resolution $=$ $3.125 \times 3.125 \mathrm{~mm}$; slice thickness $=3.0 \mathrm{~mm}$; inter-slice gap $=0.3 \mathrm{~mm}$. Using a mid-sagittal scout image, 16 axial slices were oriented in the anterior-posterior commissure (AC-PC) plane, with the lowermost slice positioned to be $20 \mathrm{~mm}$ below the AC-PC line. In addition, high-resolution, T1-weighted anatomical images of the entire brain were obtained in 3D using the mp-rage (magnetisation-prepared, rapid acquisition gradient echo) pulse sequence with the following parameters: TR $=40 \mathrm{~ms}$; TE $=5 \mathrm{~ms} ; \alpha=$ $40^{\circ} ; 1$ excitation; $\mathrm{FOV}=230 \mathrm{~mm} ;$ matrix $=256 \times 256$; 128 sagittal slices with $1.25 \mathrm{~mm}$ slice thickness.

During scanning, the room lights were dimmed and the subjects' eyes were open. Auditory stimuli were presented binaurally using a digital playback system and a magnetically shielded transducer system. The acoustic stimulation system terminated in tightly occlusive headphones allowing unimpeded conduction of the stimulus with good suppression of ambient scanner noise by about $20 \mathrm{~dB}$. During each experimental condition, a series of 95 images were acquired. Each series consisted of multiple periods of 'baseline' (OFF), during which subjects heard only the ambient machine noise, alternating with periods of 'activation' (ON), during which prepared auditory stimuli were delivered. Each series began with five baseline images (30 $\mathrm{s}$ interval), followed by 90 images during which 'rest' alternated with 'activation' every $54 \mathrm{~s}(108 \mathrm{~s} /$ cycle, 18 images/cycle, five cycles). The total duration of each image series was about 9 min.

Stimuli were 16-bit, digitally-sampled tones (pure sine waves: $1000 \mathrm{~Hz}$ ) with 200, 400, 600, or $800 \mathrm{~ms}$ duration (20 ms rise and fall time). The order of tones was randomised for each subject. The interval between tone onsets was $1 \mathrm{~s}$. Because of the limitations involved in taking an SPL meter into an MRI scanner, the intensity of the stimuli (mean signal intensity (SPL) during a 30-s epoch) was determined outside the scanner using an artificial head (Bruel and Kjaer KA637) wearing the headphones. The noise level within the MRI scanner was measured $1 \mathrm{~m}$ from the bore of the scanner magnet with a capacitance microphone revealing a rms level of 90 to $100 \mathrm{~dB}$. Because the attenuation factor of the headphones was about $20 \mathrm{~dB}$, the average intensity of the scanner noise perceived by the subjects was estimated to be about 70 to $80 \mathrm{~dB}$. Frequency analysis of the noise generated by the EPI sequence recorded in the scanner revealed five prominent frequencies. The corresponding amplitudes relative to the maximum amplitude were: $612 \mathrm{~Hz}(-15 \mathrm{~dB}), 1847 \mathrm{~Hz}$ $(-29 \mathrm{~dB}), 3036 \mathrm{~Hz}(-21 \mathrm{~dB}), 3365 \mathrm{~Hz}(-37 \mathrm{~dB})$, and $3644 \mathrm{~Hz}(-36 \mathrm{~dB})$. This analysis revealed no overlap of the prominent frequencies of the EPI-noise with the stimulus frequency of $1000 \mathrm{~Hz}$. In order to further reduce possible masking effects of scanner noise on the experimental stimuli, a TR of $6 \mathrm{~s}$ was adopted. Stimulus presentation was triggered by the EPI sequence to fall in between the interscan gap of $4 \mathrm{~s}$ (sequence scanning time $=2 \mathrm{~s})$ resulting in a non-masked presentation of the stimuli (4 stimuli per sequence).

Image analysis was performed on an Ultra 4 workstation (Sun Microsystems) using MATLAB (Mathworks, Natick, MA, USA) and SPM96b software (SPM software, MRC Cyclotron Unit, London) [14]. The first five images of each time-series, during which the MR signal reaches a steady-state, were discarded. The 90 remaining volume images of each condition were automatically realigned to the first image to correct for head movement between scans $[13,17]$. The images of the four conditions were then coregistered and spatially normalized into the Montreal Neurological Institute (MNI) template [12,34]. The procedure starts with a 12-parameter affine transformation and a six-parameter three-dimensional quadratic (or second order) deformation followed by non-linear (plastic) deformations on a slice by slice basis using Fourier-like basis functions; the parameters are estimated using a leastsquares approach after linearizing the problem [13]. In this space one pixel represents $4 \times 4 \mathrm{~mm}$ in the $x$ and $y$ dimensions, with an interplanar distance of $4 \mathrm{~mm}$. These stereotaxically transformed functional data sets from each subject were smoothed slightly with a Gaussian filter of root-mean-square radius of $8 \mathrm{~mm}$ to improve the signalto-noise ratio and to approximate normal distribution of the data. Voxels that had values greater than 0.8 of the volume mean in all the images were selected to restrict analysis to intracranial regions. The effects of global (whole volume activity) and time were removed using linear regression and sine/cosine functions as confounds (up to a maximum of 2.5 cycles per 90 scans). Removing the latter confounds corresponds to high-pass filtering the time series to remove low frequency artefacts due to cardio-respiratory and other cyclical components.

Data analysis was performed by modeling the different conditions (rest, 200, 400, 600, and $800 \mathrm{~ms}$ tone duration) as stimulus function (i.e., box car functions convolved with a hemodynamic response function) in the context of the general linear model as employed by SPM96 [15,16,36]. This analysis can be seen as a multiple linear regression (which is a special case of the general linear model) using boxcar functions of the experimental conditions (convolved with the hemodynamic function) as regressors. Task $\times$ Time interactions, that is, changes over time in the difference between activation and baseline signal, were also modeled as effects of interest, using a set of polynomial basis functions (up to the third order). In addition, planned comparisons between the four conditions were performed. These effects were tested by applying appropriate linear contrasts to the parameter estimates for each condition resulting in a $t$ statistic for each and every voxel. These $t$-statistics (transformed to $Z$-statistics) constitute a statistical parametric map (SPM). The resulting SPM was then thresholded at $p<0.01$ (3D corrected for multiple comparisons). The activated voxels surviving this procedure were superimposed on 'SPM brain projections' and 
on individual stereotaxically normalised high-resolution MR-anatomical scans (individual analysis). In addition, the same statistic was performed for the group data (group analysis). The thresholded voxels for the group analysis were also superimposed on 'SPM brain projections' and on the mean high-resolution MRI. For the primary and secondary auditory cortex, the mean time course of activation was plotted by considering the adjusted data of the peak activation. The applied spatial smoothing of the data set means that the signal change within a given voxel represents the average signal change in a small region around that voxel, weighted by the smoothing kernel employed ( 8 $\mathrm{mm}$ in all three dimensions).

Because the clusters of activated voxels covered a wide range of the auditory cortex, we additionally defined regions of interest (ROI) in each hemisphere to account for the diversity of the auditory cortex (primary, immediate, and secondary auditory cortex). These ROIs were defined according to the Talairach atlas and prominent sulcal and gyral landmarks (Heschl's gyrus, sylvian fissure, ramus posterior ascendens, ramus posterior descendens, and superior temporal sulcus) $[18,19,23,32]$ : (1) the primary and immediate auditory area (Brodmann area, BA 41/42) and (2) the auditory association area (BA 22). However, the exact spatial delineation of the auditory cortices is currently not possible for the following reasons: (i) the spatial extent of the cytoarchitectonic area of the primary and immediate auditory cortex is currently unknown, (ii) the exact delineation of the anatomical landmarks was not possible because of the limited spatial resolution of the normalized fMRI images $\left(4 \mathrm{~mm}^{3}\right)$, (iii) the angulated shape of the Heschl's gyri and the sylvian fissure varied considerably in horizontal and vertical directions within subjects (left vs. right hemisphere) and between subjects. Thus, we defined as primary and immediate auditory cortex a region covering the Heschl's gyrus (as defined by the Heschl's sulcus and the first transverse sulcus) on at least two horizontal planes taken from the Talairach atlas $(z=12$ and $z=8$ ). The secondary auditory cortex was defined as the area posterior to the primary and immediate auditory cortex extending into the inferior and superior directions from plane $z=0$ and to $z=20$ more posteriorly. Thus, our ROI definitions and BA designations should be taken only as approximate. For these ROIs adjusted BOLD signal changes for the voxels of peak activation in right and left primary and secondary auditory cortex are plotted in Figs. 2 and 3. Inspection of other voxels from the same ROIs (data not shown) showed the same relationship be-

\section{$200 \mathrm{~ms}$}

$400 \mathrm{~ms}$
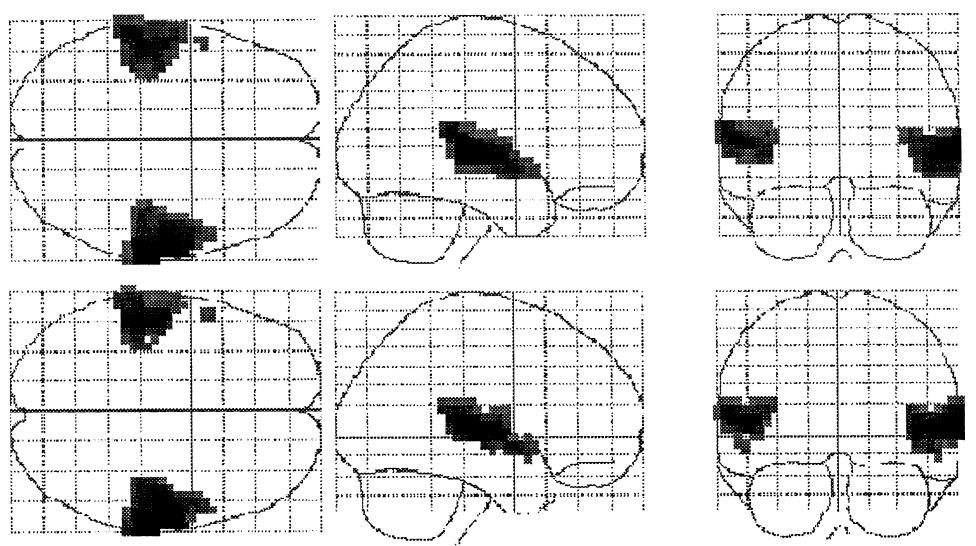

$600 \mathrm{~ms}$
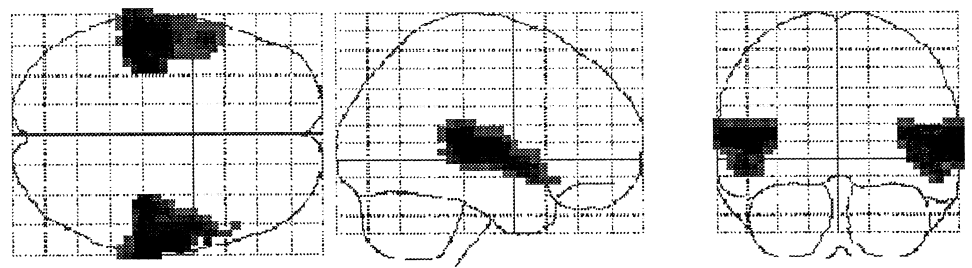

$800 \mathrm{~ms}$
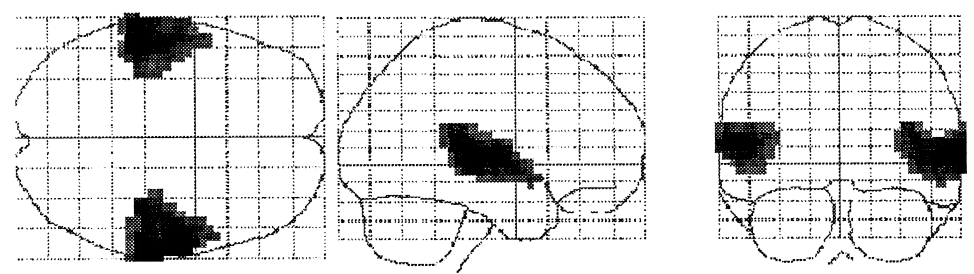

Fig. 1. The three brain projections viewing the brain from above (transverse), the right (sagittal), and the back (coronal) illustrate the regions of significant activation vs. rest differences for the four stimulation conditions $(p<0.01,3 \mathrm{D}$ corrected). 
Table 1

Location of STG (superior temporal gyrus) activation in the right (R) and left (L) hemisphere for the group analysis according to the Talairach and Tournoux atlas [34]

\begin{tabular}{|c|c|c|c|c|c|c|}
\hline Condition & $\begin{array}{l}\text { Anatomical } \\
\text { regions }\end{array}$ & $Z$ statistic & $\begin{array}{l}N \text { of } \\
\text { voxel }\end{array}$ & $x$ & $y$ & $z$ \\
\hline \multirow[t]{3}{*}{$200 \mathrm{~ms}$} & $\mathrm{R} \mathrm{STG}^{\mathrm{a}}$ & 8.59 & 265 & 64 & -28 & 8 \\
\hline & & $8.13^{\mathrm{b}}$ & & 56 & -12 & 4 \\
\hline & $\mathrm{L} \mathrm{STG}^{\mathrm{a}}$ & 7.81 & 178 & -56 & -28 & 12 \\
\hline \multirow[t]{5}{*}{$400 \mathrm{~ms}$} & $\mathrm{R} \mathrm{STG}^{\mathrm{a}}$ & 8.61 & 242 & 68 & -24 & 4 \\
\hline & & $8.26^{\mathrm{b}}$ & & 56 & -16 & 8 \\
\hline & & $7.35^{\mathrm{b}}$ & & 44 & -28 & 12 \\
\hline & $\mathrm{LSTG}^{\mathrm{a}}$ & 7.93 & 149 & -48 & -32 & 12 \\
\hline & & $7.85^{\mathrm{b}}$ & & -52 & -16 & 8 \\
\hline \multirow[t]{6}{*}{$600 \mathrm{~ms}$} & $\mathrm{R} \mathrm{STG}^{\mathrm{a}}$ & 8.69 & 288 & 64 & -28 & 8 \\
\hline & & $8.24^{\mathrm{b}}$ & & 60 & -12 & 8 \\
\hline & & $7.97^{b}$ & & 48 & -28 & 12 \\
\hline & $\mathrm{LSTG}^{\mathrm{a}}$ & 8.50 & 277 & -52 & -28 & 12 \\
\hline & & $8.17^{\mathrm{b}}$ & & -52 & -16 & 8 \\
\hline & & $7.55^{\mathrm{b}}$ & & -40 & -20 & 12 \\
\hline \multirow[t]{5}{*}{$800 \mathrm{~ms}$} & $\mathrm{R} \mathrm{STG}^{\mathrm{a}}$ & 8.65 & 318 & 68 & -24 & 4 \\
\hline & & $8.35^{\mathrm{b}}$ & & 56 & -12 & 4 \\
\hline & & $7.83^{\mathrm{b}}$ & & 40 & -28 & 16 \\
\hline & $\mathrm{LSTG}^{\mathrm{a}}$ & 8.37 & 219 & -52 & -28 & 12 \\
\hline & & $8.00^{\mathrm{b}}$ & & -52 & -16 & 8 \\
\hline
\end{tabular}

${ }^{\mathrm{a}}$ The reported clusters comprise activated voxels within the primary and secondary auditory cortex as well as voxels from the auditory association cortex.

${ }^{\mathrm{b}}$ Further local maxima belonging to the cluster represented by the largest $Z$-score. tween the BOLD response and stimulus duration. However, with that paper, we are only reporting the time course of the peak activations.

\section{Results}

Comparison of each auditory activation condition compared to rest produced highly significant bilateral increases in the BOLD signal involving the primary and secondary auditory cortex on the superior temporal gyrus (Fig. 1 and Table 1). The planned comparisons between the activations obtained in the four auditory stimulation conditions revealed no significant difference. There was also no significant Time $\times$ Task interaction. Thus, there was no effect of stimulus duration on the location, intensity, as well as the spatial extent of activation within the primary and secondary auditory cortex.

In order to compare the time courses obtained for the different stimulus durations, we performed for each region of interest a regression analysis modelling duration (applying effect coding) and sequence number (applying polynomials up to the eight order) as predictors and the BOLD response as dependent variable. The repeated measurement effect was partialised out according to the recommendations given by Pedhazur [28]. Applying these statistics, we

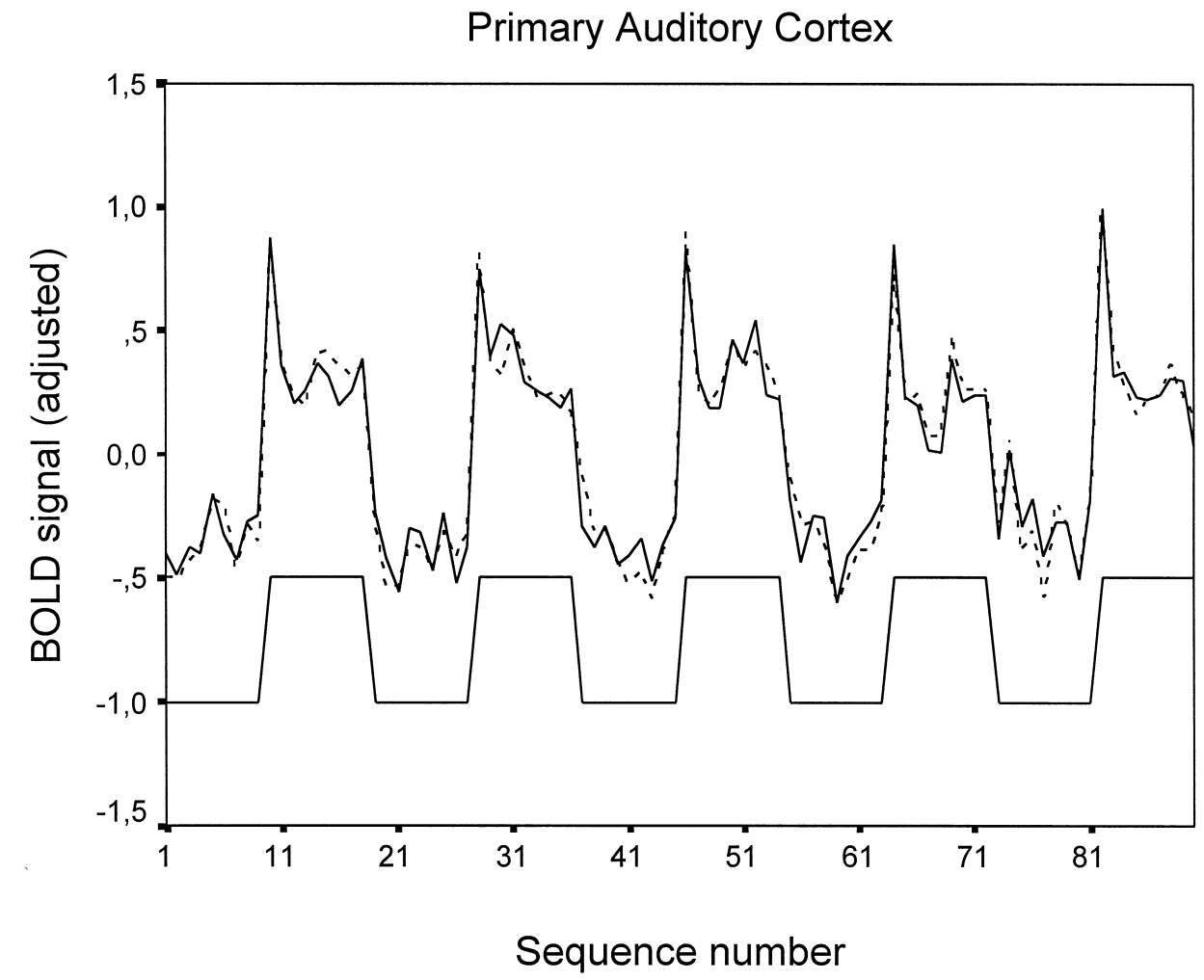

Fig. 2. Time course of the mean BOLD signal from the significantly activated voxels in the primary auditory cortex in the left (dashed line) and right (solid line) hemisphere. 


\section{Secondary Auditory Cortex}

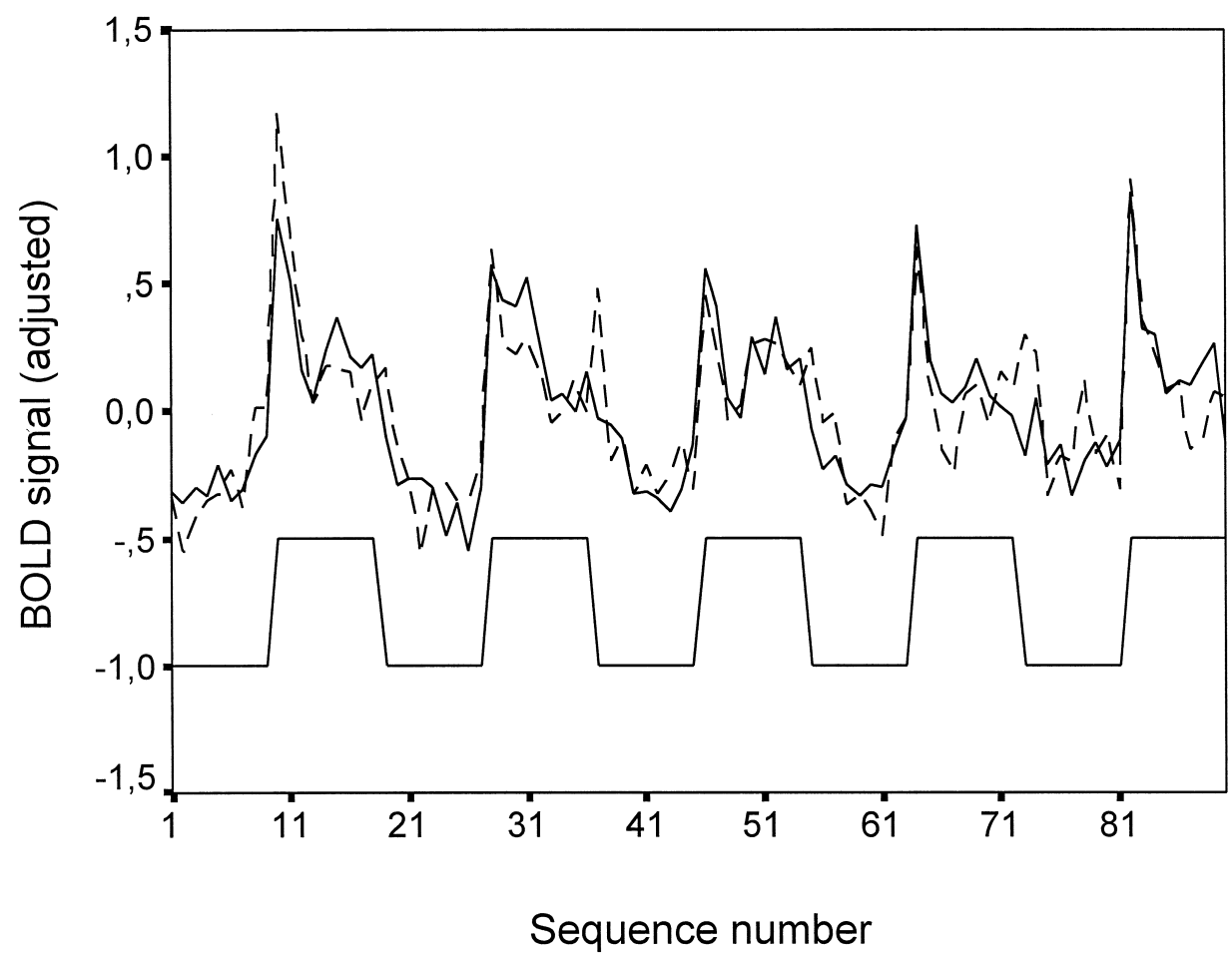

Fig. 3. Time course of the mean BOLD signal from the significantly activated voxels in the secondary auditory cortex in the left (dashed line) and right (solid line) hemisphere.

found no time course differences due to stimulus duration. Therefore, these time courses were averaged across all stimulus durations. These mean time courses averaged across all subjects are depicted in Figs. 2 and 3. From these time courses, one can easily identify that the first response of each ON period is the largest and that the following responses are gradually decreasing. In order to demonstrate this statistically, we calculated for each subject an average $\mathrm{ON}$ period. The nine mean signals were then subjected to a nine-way repeated measurement analysis of variance with sequence number as factor (Figs. 4 and 5 are demonstrating these means). Each ANOVA revealed

\section{Primary Auditory Cortex}

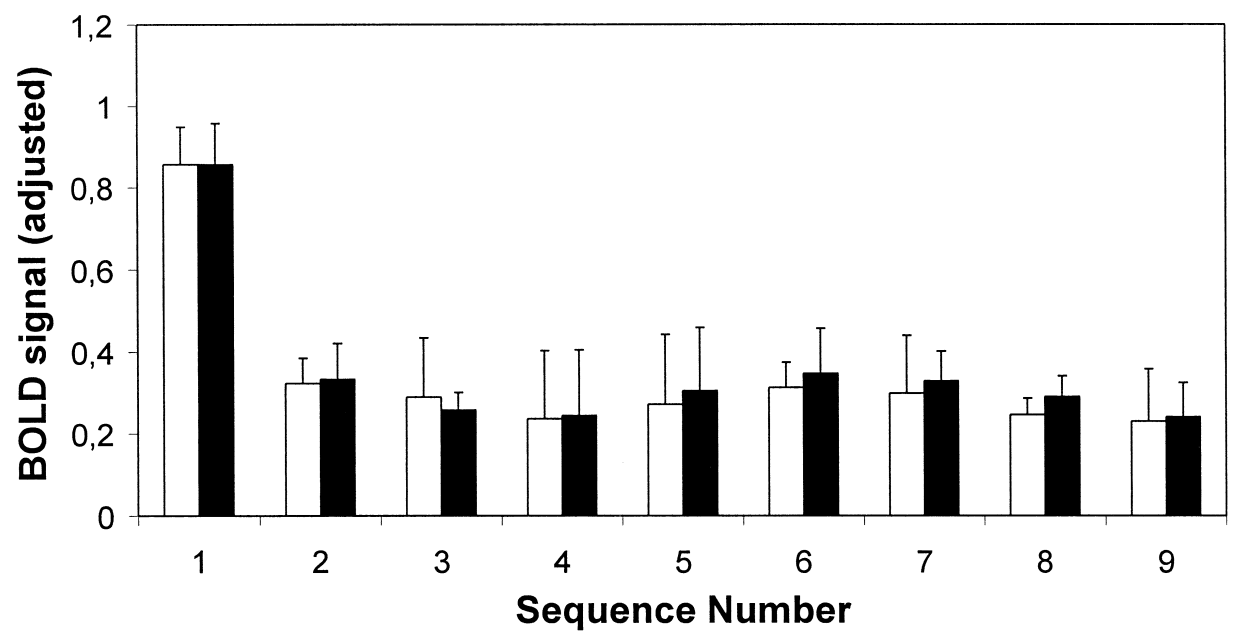

Fig. 4. Mean BOLD signals broken down by sequence number for the primary auditory cortex. Open bars representing the means for the left primary auditory cortex, and filled bars representing the means for right primary auditory cortex. Standard deviations are indicated as vertical lines. 


\section{Secondary Auditory Cortex}

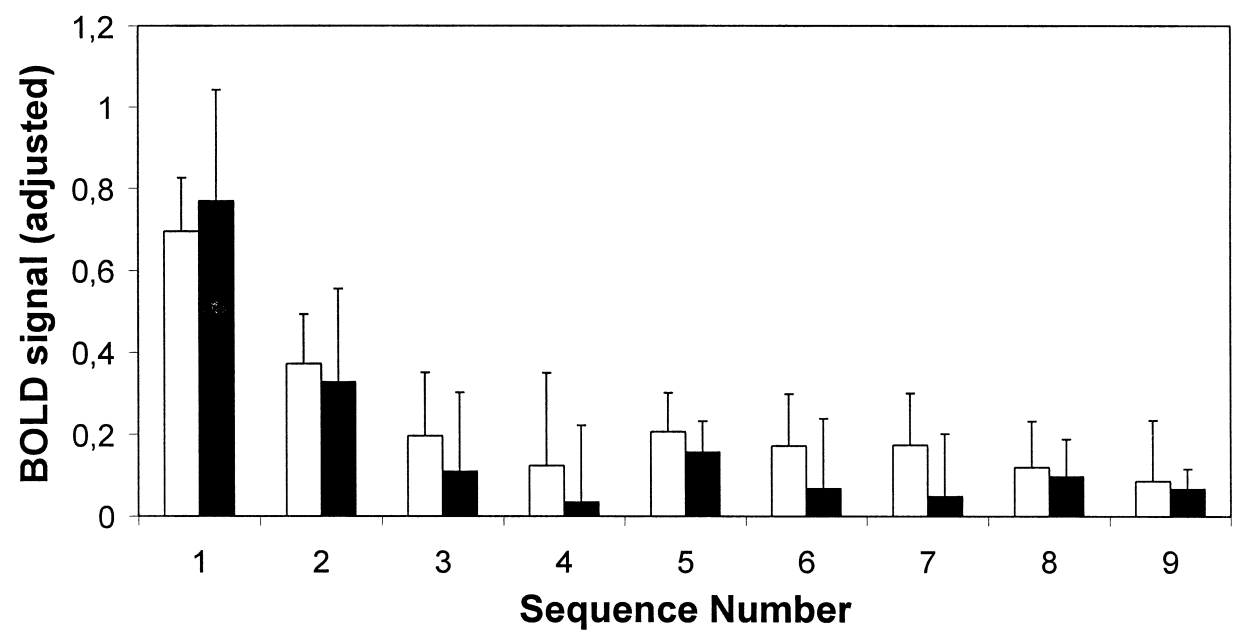

Fig. 5. Mean BOLD signals broken down by sequence number for the secondary auditory cortex. Open bars representing the means for the left secondary auditory cortex, and filled bars representing the means for right secondary auditory cortex. Standard deviations are indicated as vertical lines.

a significant main effect of sequence number (all $p$-values $<0.001)$. Subsequent planned contrasts revealed that constantly, the first sequence came up with the largest BOLD signal (all $p$-values $<0.001$ ). The following signals did not differ significantly from each other (all $p$-values at least $>0.10$ ).

\section{Discussion}

The present results demonstrate that tone duration does not have an inevitable substantial influence on the hemodynamic response in the auditory cortex at least for tone durations and the presentation rate applied here. This is quite astonishing considering the strong difference in the integrated stimulus duration for each scanning sequence. In effect, during the $800 \mathrm{~ms}$ condition, the integrated stimulus duration is $3200 \mathrm{~s}$ while for the $200 \mathrm{~ms}$ condition, the integrated stimulus duration is $800 \mathrm{~ms}$. In other words, for the $800 \mathrm{~ms}$ condition, the auditory stimulus is present during $80 \%$ of the $4 \mathrm{~s}$ lasting between scan time (effective $\mathrm{TR}=6 \mathrm{~s}$, scanning time $=2 \mathrm{~s}$, masking free presentation period $=4 \mathrm{~s}$ ) while during the $200 \mathrm{~ms}$ condition, the auditory stimulus is only present during $20 \%$ of the presentation period. Although the integrated stimulus duration is four times longer for the $800 \mathrm{~ms}$ condition than for the $200 \mathrm{~ms}$ condition, there is no difference in the hemodynamic response in the auditory cortex.

As shown in our own experiment, it may be possible that the relatively slow hemodynamic response is most strongly driven by stimulus onsets than by steady state aspects. Similar results demonstrating stronger hemodynamic responses during the first scans in fMRI experiments have been reported by two previously published studies using auditory speech stimuli or flashing light [9], thus corroborating our own results [27]. The possible reasons for this time course are currently unknown, however, several factors may be involved, including top-down mechanisms and/or the interplay of tissue perfusion and oxygen consumption. Which of these mechanisms may actually be responsible for the pattern of hemodynamic response found in our study has to be elucidated in future studies. Nevertheless, this time course remarkably resembles typical time courses of neural activation found in human auditory ERP studies, demonstrating strong ON and OFF responses to tone onsets and/or cessations [2,31]. This pattern of neural activity may reflect the predominant property of cortical neurons in the auditory cortex which are not generally sensitive to an invariant long-duration stimulus. Most auditory neurons respond to a sudden change in intensity (to the transient onset or offset of a tone or noise burst) $[1,5,21,22]$. However, beside these neurophysiological interpretations, one has also to consider the fact that the experimental paradigm applied here does not invoke further psychological processing of the stimuli (categorization, attentive monitoring, etc.). Thus, it seems likely that only the automatic processes and the concomitant neural activation for analysing auditory stimuli are evoked. These automatic processes might comprise the basic frequency and intensity analysis within the primary and secondary auditory cortex. It might be possible that within the context of different experimental paradigms in which further processing of auditory stimuli are evoked, a duration effect and a stronger tonic phase of the hemodynamic response might be uncovered.

It might also be possible that the time courses found in our study are reflecting the sensory adaptation occurring at the receptor level as a function of stimulation fatigue, 
which makes the receptor refractory (insensitive) to new stimulation. This mechanism should not be confused with the 'orienting-habituation' cycle, probably involving cortical processing [30]. This latter mechanism is characterized by the fact that the first presentation of a stimulus is accompanied by the orienting response (OR). This OR is a kind of 'surprise'-reaction and it involves a shift of attention toward the new and mostly unexpected stimulus. The OR consists of sensory, somatic, EEG, and autonomic changes, which together interrupt ongoing behavior and increase sensory acuity. In other words, the OR facilitates sensory input to the brain. In terms of informationprocessing models, the OR reflects passive attention to stimulus input that is amplified in the nervous system until it interrupts ongoing activity. At this stage of information processing incoming stimuli are enhanced and elicit enhanced neural responses [25,26,35]. After repeated presentations of the same stimulus, the OR becomes smaller and smaller, and finally disappears. An interesting observation in this respect is that the OR will be reinforced after the omission of the stimulus, because the sequence is disrupted.

The findings reported here should prove useful as an initial guide in future experiments because we demonstrated that stimulus duration of auditory stimuli do not have an inevitable influence on the BOLD response in the primary and secondary auditory cortex. This result is especially important for designing studies applying language stimuli (words or syllables) which considerably vary with respect to stimulus duration. In addition, many linguistic and phonetic aspects are coded in terms of durational characteristics, for instance, rate of change of formant transitions or voice onset times. As a general rule regarding speech stimuli, it seems advisable to use stimuli durations in a range of 200 to $800 \mathrm{~ms}$ in duration without confounding the results with a general duration effect.

\section{Acknowledgements}

This study was financed by the Deutsche Forschungsgemeinschaft (DFG JA 737/5-1). The guest scientists (TWB, $\mathrm{KL}, \mathrm{LJ})$ would like to thank the Research Centre Jülich for providing the facilities that made this work possible. Tony Buchanan was supported by a grant from the Research Centre Jülich as well as an individual National Research Service Award (\# 1 F31 MH 11844-01) from the National Institute of Mental Health, USA.

\section{References}

[1] M. Abeles, M.H.J. Goldstein, Responses of single units in the primary auditory cortex of the cat to tones and to tone pairs, Brain Res. 42 (1972) 337-352.

[2] V.N. Andreeva, Y.G. Kratin, S. Kurbanov, Auditory stimulus dura- tion effects on discrimination and brain activity, Neurosci. Behav. Physiol. 5 (1972) 10-23.

[3] P.A. Bandettini, A. Jesmanowicz, J. Van Kylen, R.M. Birn, J.S. Hyde, FMRI of scanner noise induced auditory cortex activation, Proceedings of the International Society of Magnetic Resonance in Medicine, 1997, 349 (Abstract).

[4] P.A. Bandettini, A. Jesmanowicz, J. Van Kylen, R.M. Birn, J.S. Hyde, Functional MRI of brain activation induced by scanner acoustic noise, Magn. Reson. Med. 39 (1998) 410-416.

[5] D.A. Benson, R.D. Hienz, M.H.J. Goldstein, Single-unit activity in the auditory cortex of monkeys actively localizing sound sources: spatial tuning and behavioral dependency, Brain Res. 219 (1981) 249-267.

[6] J.R. Binder, Neuroanatomy of language processing studied with functional MRI, Clin. Neurosci. 4 (1997) 87-94.

[7] J.R. Binder, J.A. Frost, T.A. Hammeke, R.W. Cox, S.M. Rao, T. Prieto, Human brain language areas identified by functional magnetic resonance imaging, J. Neurosci. 17 (1997) 353-362.

[8] J.R. Binder, J.A. Frost, T.A. Hammeke, S.M. Rao, R.W. Cox, Function of the left planum temporale in auditory and linguistic processing, Brain 119 (1996) 1239-1247.

[9] J.R. Binder, S.M. Rao, T.A. Hammeke, J.A. Frost, P.A. Bandettini, J.S. Hyde, Effects of stimulus rate on signal response during functional magnetic resonance imaging of auditory cortex, Cognit. Brain Res. 2 (1994) 31-38.

[10] J.R. Binder, S.M. Rao, T.A. Hammeke, J.A. Frost, P.A. Bandettini, A. Jesmanowicz, J.S. Hyde, Lateralized human brain language systems demonstrated by task subtraction functional magnetic resonance imaging, Arch. Neurol. 52 (1995) 593-601.

[11] J.R. Binder, S.M. Rao, T.A. Hammeke, F.Z. Yetkin, A. Jesmanowicz, P.A. Bandettini, E.C. Wong, L.D. Estkowski, M.D. Goldstein, V.M. Haughton et al., Functional magnetic resonance imaging of human auditory cortex [see comments], Ann. Neurol. 35 (1994) 662-672.

[12] A.C. Evans, D.L. Collins, S.R. Mills, E.D. Brown, R.L. Kelly, T.M. Peters, 3D statistical neuroanatomical models from 305 MRI volumes, Proc. IEEE-Nuclear Science Symposium and Medical Imaging, 1993, pp. 1813-1817.

[13] K.J. Friston, J. Ashburner, J.B. Poline, C.D. Frith, J.D. Heather, R.S.J. Frackowiack, Spatial realignement and normalization of images, Hum. Brain Mapp. (1997).

[14] K.J. Friston, A.P. Holmes, J. Ashburner, J.B. Poline, World Wide Web http://www.fil.ion.ucl.ac.uk/spm., 1996.

[15] K.J. Friston, A.P. Holmes, J.B. Poline, P.J. Grasby, S.C.R. Williman, R.S.J. Frackowiack, R. Turner, Analysis of fMRI time-series revisited, Neuroimage 2 (1995) 45-53.

[16] K.J. Friston, A.P. Holmes, K.P. Worsley, J.-B. Poline, C.D. Frith, R.S.J. Frackowiack, Statistical parametric maps in functional imaging: a general linear approach, Hum. Brain Mapp. 2 (1995) 189-210.

[17] K.J. Friston, S. Williams, R. Howard, R.S. Frackowiak, R. Turner, Movement-related effects in fMRI time-series, Magn. Reson. Med. 35 (1996) 346-355.

[18] A.M. Galaburda, F. Sanides, Cytoarchitectonic organization of the human auditory cortex, J. Comp. Neurol. 190 (1980) 597-610.

[19] A.M. Galaburda, F. Sanides, N. Geschwind, Human brain: cytoarchitectonic left-right asymmetries in the temporal speech region, Arch. Neurol. 35 (1978) 812-817.

[20] B. Gaschler-Markefski, F. Baumgart, C. Tempelmann, F. Schindler, D. Stiller, H.J. Heinze, H. Scheich, Statistical methods in functional magnetic resonance imaging with respect to nonstationary timeseries: auditory cortex activity, Magn. Reson. Med. 38 (1997) 811-820.

[21] M.H.J. Goldstein, M. Abeles, Note on tonotopic organization of primary auditory cortex in the cat, Brain Res. 100 (1975) 188-191.

[22] M.H.J. Goldstein, J.L. Hall, B.O. Butterfield, Single-unit activity in the primary auditory cortex of unanesthetized cats, J. Acoust. Soc. Am. 43 (1968) 444-455. 
[23] L. Jäncke, G. Schlaug, Y. Huang, H. Steinmetz, Asymmetry of the planum parietale, NeuroReport 5 (1994) 1161-1163.

[24] L. Jäncke, N.J. Shah, S. Posse, M. Grosse-Ryuken, H.W. MullerGartner, Intensity coding of auditory stimuli: an fMRI study, Neuropsychologia 36 (1998) 875-883.

[25] L. Jäncke, S. Mirzazade, N.J. Shah, Attention modulates activity in the primary and the secondary auditory cortex: a functional magnetic resonance imaging study, Neurosci. Lett. 266 (1999) 125-128.

[26] L. Jäncke, S. Mirzazade, N.J. Shah, Attention modulates the blood oxygen level dependent response in the primary visual cortex measured with functional magnetic resonance imaging, Naturwissenschaften 86 (1999) 79-81.

[27] A. Kleinschmidt, O. Gruber, S. Posse, L. Jäncke, M. Grosse-Ruyken, H.-W. Müller-Gärtner, H.-J. Freund, Deriving stimulus-response functions from cerebral blood oxygenation-optimizing timing and design of sensory activation paradigms, Neuroimage 5 (1997) S38, Abstract.

[28] E.J. Pedhazur, Multiple Regression in Behavioral Research: Explanation and Prediction, Holt, Rinehart and Winston, New York, 1982.

[29] H. Scheich, F. Baumgart, B. Gaschler-Markefski, C. Tegeler, C. Tempelmann, H.J. Heinze, F. Schindler, D. Stiller, Functional magnetic resonance imaging of a human auditory cortex area involved in foreground-background decomposition, Eur. J. Neurosci. 10 (1998) 803-809.
[30] D.A. Siddle, Orienting, habituation, and resource allocation: an associative analysis, Psychophysiology 28 (1991) 245-259.

[31] M. Spreng, W.D. Keidel, Separation of the cerebro-audiogram (CAG), neuro-audiogram (NAG) and oto-audiogram (OAG) in the objection audiometry, Arch. Klin. Exp. Ohren-, Nasen-Kehlkopfheilkd. 189 (1967) 225-246.

[32] H. Steinmetz, J. Rademacher, L. Jancke, Y.X. Huang, A. Thron, K. Zilles, Total surface of temporoparietal intrasylvian cortex: diverging left-right asymmetries, Brain Lang. 39 (1990) 357-372.

[33] D. Stiller, B. Gaschler-Markefski, F. Baumgart, F. Schindler, C. Tempelmann, H.J. Heinze, H. Scheich, Lateralized processing of speech prosodies in the temporal cortex: a 3-T functional magnetic resonance imaging study, MAGMA 5 (1997) 275-284.

[34] J. Talairach, P. Tournoux, Co-Planar Stereotaxic Atlas of the Human Brain, 3-Dimensional Proportional System: An Approach to Cerebral Imaging, Thieme Medical Publishers, New York, 1988.

[35] M.G. Woldorff, C.C. Gallen, S.A. Hampson, S.A. Hillyard, C. Pantev, D. Sobel, F.E. Bloom, Modulation of early sensory processing in human auditory cortex during auditory selective attention, Proc. Natl. Acad. Sci. USA 90 (1993) 8722-8726.

[36] K.J. Worsley, K.J. Friston, Analysis of fMRI time-series revisitedagain, Neuroimage 3 (1995) 173-181. 